
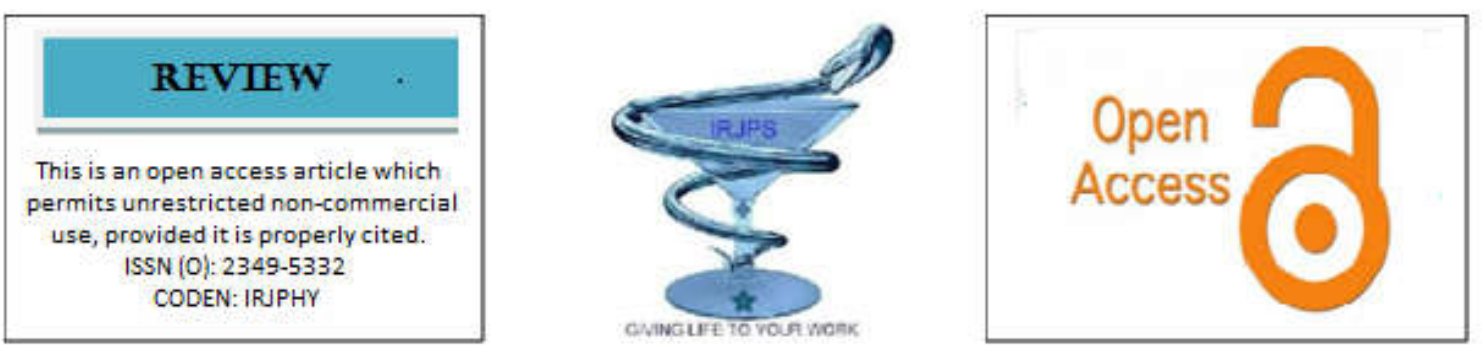

\title{
COVID-19: NEW PANDEMIC SITUATION ON EARTH
}

\author{
C. Mohan ${ }^{1}$, M. Verma ${ }^{2}$, M. Mittal ${ }^{3}$, R. Kumar ${ }^{3}$, V. Kumar ${ }^{3}$ *
}

$\mathrm{SBAS}^{1}$, $\mathrm{SOET}^{2}$, $\mathrm{SMAS}^{3}$, K. R. Mangalam University, Sohna Road, Gurugram-122103, India

\begin{abstract}
After more than 100 years in 1918 influenza pandemic now we face another pandemic situation currently designated as severe acute respiratory syndrome (SARS)-CoV-2, spreading to every continent on earth, forcing us to live with this virus for perhaps a long time. It was firstly identified in 2019 in Wuhan city of China and in 2020 scientist coined it as COVID- 19 very soon it infected lots of people worldwide and leading to several deaths globally and still going on. The period from the onset of COVID-19 symptoms to death ranged from 6 to 41 days with a median of 14 days, characterized by fever, cough and fatigue, while other symptoms include sputum production, headache and pneumonia. However, people of week immunity are on very high risk of this infection, but pregnant women showed same clinical characteristic of COVID-19 as of non-pregnant adult patients. In this current review, we summarize the comparison between COVID-19 and SARS- CoV, symptoms in adults and in pregnant women, clinical findings, and worldwide scenario and discuss the approaches going on to treat this infection.
\end{abstract}

KEY WORDS: COVID-19, Wuhan city, Pandemic situation, Coronavirus

Corresponding Author: V. Kumar

E-mail: vinod.kumar@,krmangalam.edu.in
Indian Research Journal of Pharmacy and Science; 25(2020)2146-2153;

Journal Home Page: https://www.irjps.in DOI: 10.21276/irjps.2020.7.2.6 


\section{INTRODUCTION}

In December 2019, unfortunately numbers of patients were identified with respiratory syndrome with unknown cause in Wuhan, Hubei Province, China followed by an outbreak across Hubei Province. After deep study scientist were identified the cause of this pandemic disease was a novel virus, named Wuhan coronavirus $(\mathrm{CoV})$, and later on International Committee on Taxonomy of Viruses (ICTV) coined its COVID-19. In a very short time this virus infected a large population worldwide and the ongoing outbreak has been declared by WHO as a global public health emergency. After more than 100 years in 1918 influenza pandemic now we face another pandemic situation currently designated as severe acute respiratory syndrome (SARS)-CoV-2, spreading to every continent on earth, forcing us to live with this virus for perhaps a long time. This novel virus was initially identified as type of common virus that infects humans, typically leading to an upper respiratory infection (URI), belonging to the family Coronaviridae possible zoonotic origin ${ }^{1}$.

Till date the, detailed morphology and ultrastructure of this virus remains incompletely understood. However, scientist believes that corona viruses has enveloped virions of approximately $120 \mathrm{~nm}$ in diameter, club-shaped glycoprotein spikes in the envelope enable it a crown-like, or coronal, appearance. The nucleocapsid, made up of a protein shell known as a capsid and containing the viral nucleic acids, is helical or tubular (Fig 1). The coronavirus genome consists of a single strand of positive-sense RNA (ribonucleic acid), genome size ranges from approximately 27 to 34 kilo bases. This genome size is one of the largest among RNA viruses. The genome has a $5^{\prime}$ methylated cap and a $3^{\prime}$ polyadenylated tail, as per the data obtained from $\mathrm{ICMR}^{2}$, India detecting the SARS-CoV-2 virus using TEM directly in a throat swab specimen confirmed by PCR.

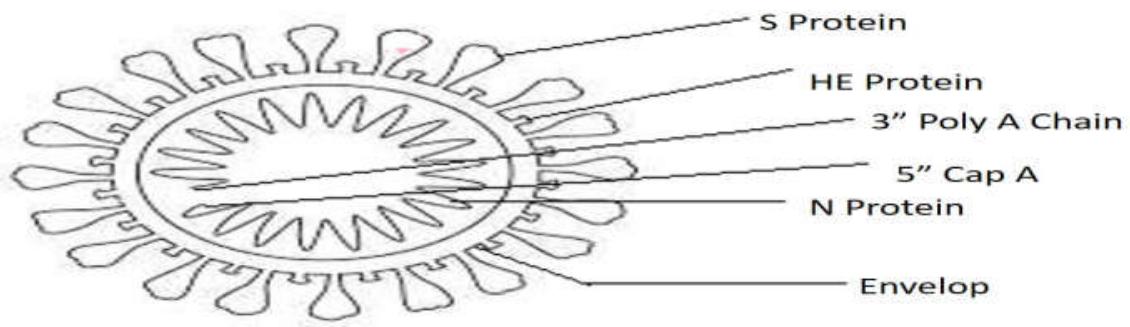

Fig 1: structure of COVID-19

In humans COVID-19 infection characterized by upper respiratory tract infections that can be mild, such as some cases of the common cold (among other possible causes, predominantly rhinoviruses), and others that can be lethal, such as SARS, MERS, and COVID-19, however other species showed various types of symptoms like in chickens, they cause an upper respiratory tract disease, while in cows and pigs they cause diarrhea ${ }^{3}$.

The first human corona viruses were discovered in the late 1960's afterword in May 2003, another strain of coronavirus SARS was identified in wild animals sold in local food market of Guangdong, China and in year 2004 in Nederland HCoV-NL63 was identified, and in year 2019 we face new strain of corona virus named COVID-19 again in China which infected very large population worldwide and leading to several deaths worldwide and still going on as shown in (Fig 2).

A recent study stated that around $3.84 \%$ of total cases are of healthcare professionals were infected in Wuhan city, around $41 \%$ patients were having hospital related problem, $21 \%$ patients were admitted in ICU and mortality rate was $4.3 \%$. Critically ill patients were having on high risk and required ventilators, such condition occurred due to presymptomatic transmission such as family and social gathering, travelling etc. clinically finding stated that the age group of 30 to 79 accounting around $86.6 \%$ of total cases and people who are traveller are on high risk of infection than others. According to recent findings, researchers can be divided SARSCoV-2 infection in to 3 stages: Stage I, asymptomatic incubation with or without detectable virus; Stage II, non-severe symptomatic period with virus; Stage III, severe respiratory symptomatic stage with high viral load. 


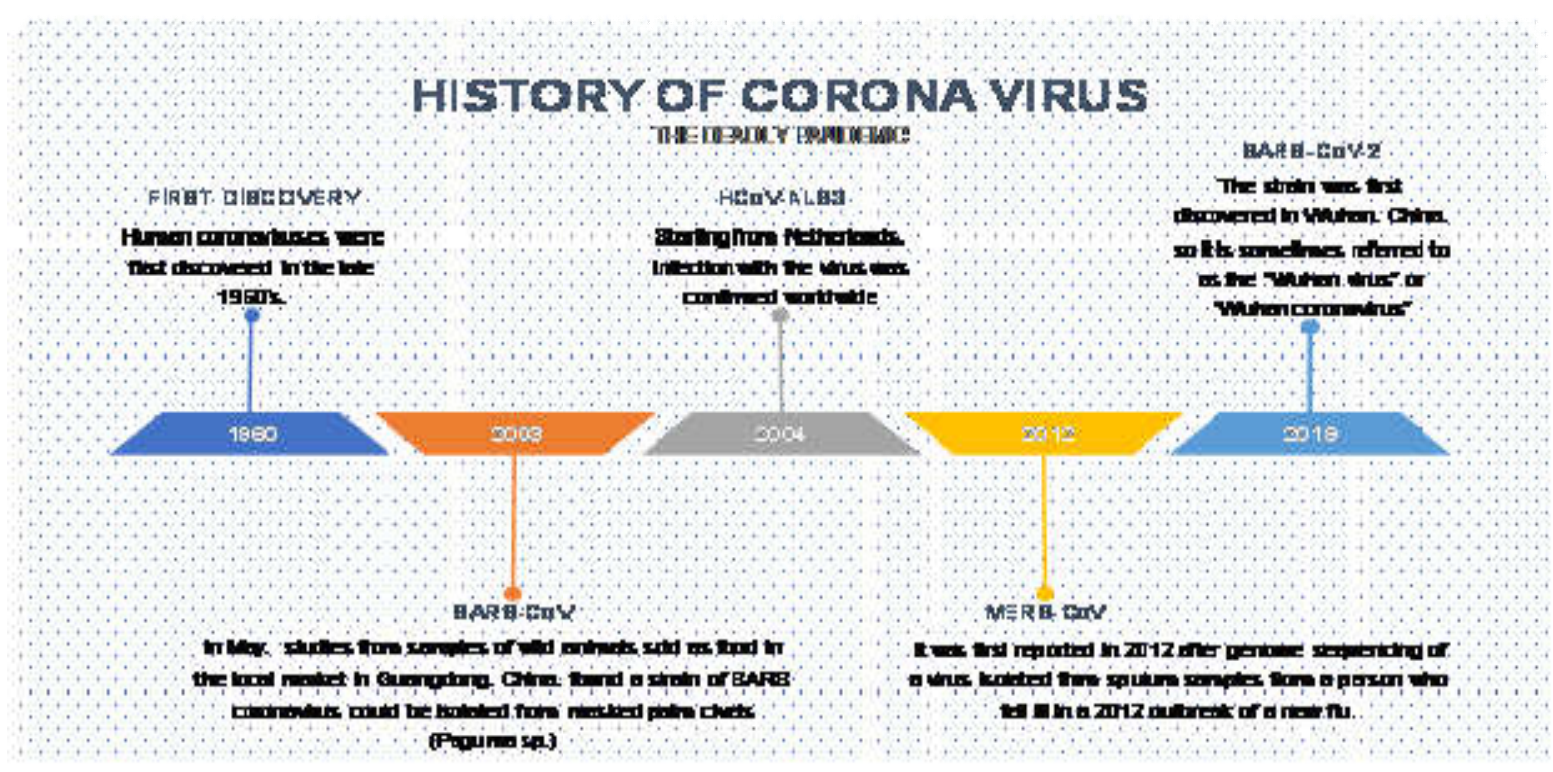

Fig 2: History of corona virus from 1960 to till date

\section{COMPARISON BETWEEN COVID-19 AND SARS-COV}

COVID-19 and SARS-CoV belongs to the same beta coronavirus subgroup, however there are many types of coronavirus (Human coronavirus, Murine coronavirus, Porcine coronavirus, Avian coronavirus, Feline coronavirus and Bovine coronavirus) similarity at

OOVID-19

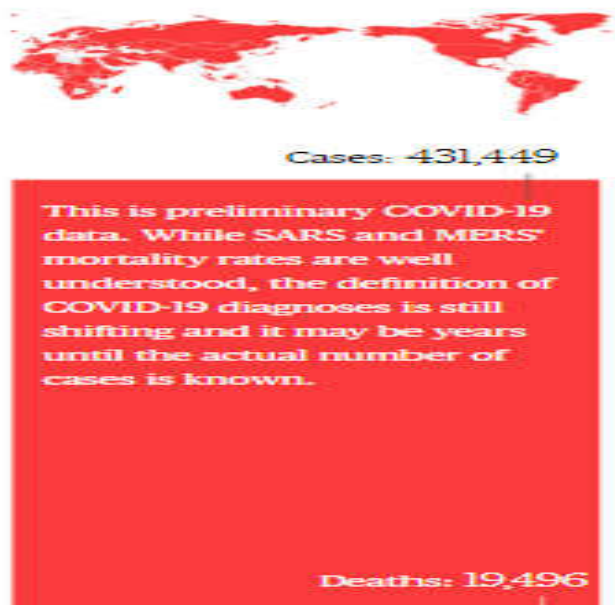

genome level is only $70 \%$, and the novel group has been found to show genetic differences from SARSCoV. Recent clinically finding stated that the Peak Period of SARS is January to February in 2003 and Peak Period of COVID 19 also January to February 2020 and comparison between the mortality rates is shown in (Fig 3).

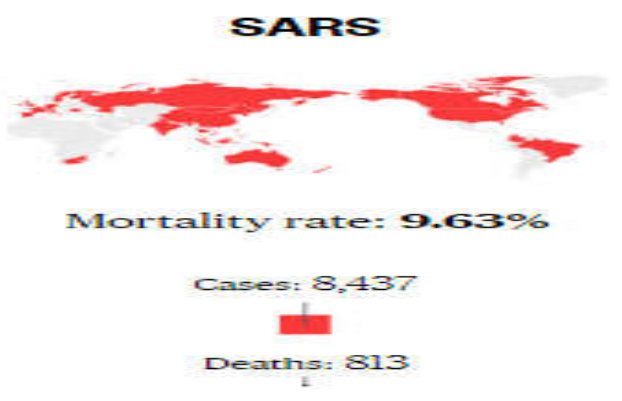

Fig 3: comparison of mortality rate between COVID 19 and SARS 


\section{Symptoms of COVID-19}

The period from the onset of COVID-19 symptoms to death ranged from 6 to 41 days with a median of 14 days. This period is dependent on the age of the patient and status of the patient's immune system. But individual variations in the immune response against pathogens leading a massive destruction of the affected tissues will occur, especially high ACE2 expression tissues, such as intestine and kidney. The damaged cells induced inflammation in the lungs leading to lifethreatening respiratory disorders at the severe stage. The most common symptoms at onset of COVID-19 illness are fever, cough and fatigue, while other symptoms include sputum production, headache, haemoptysis ${ }^{4}$, diarrhoea, dyspnoea, and lymphopenia (Fig 4). Other, less common symptoms have included headache and sore throat. In addition to respiratory symptoms, gastrointestinal symptoms (ex. nausea and diarrhoea) have also been reported.

\section{COVID-19 SYMPTOMS}

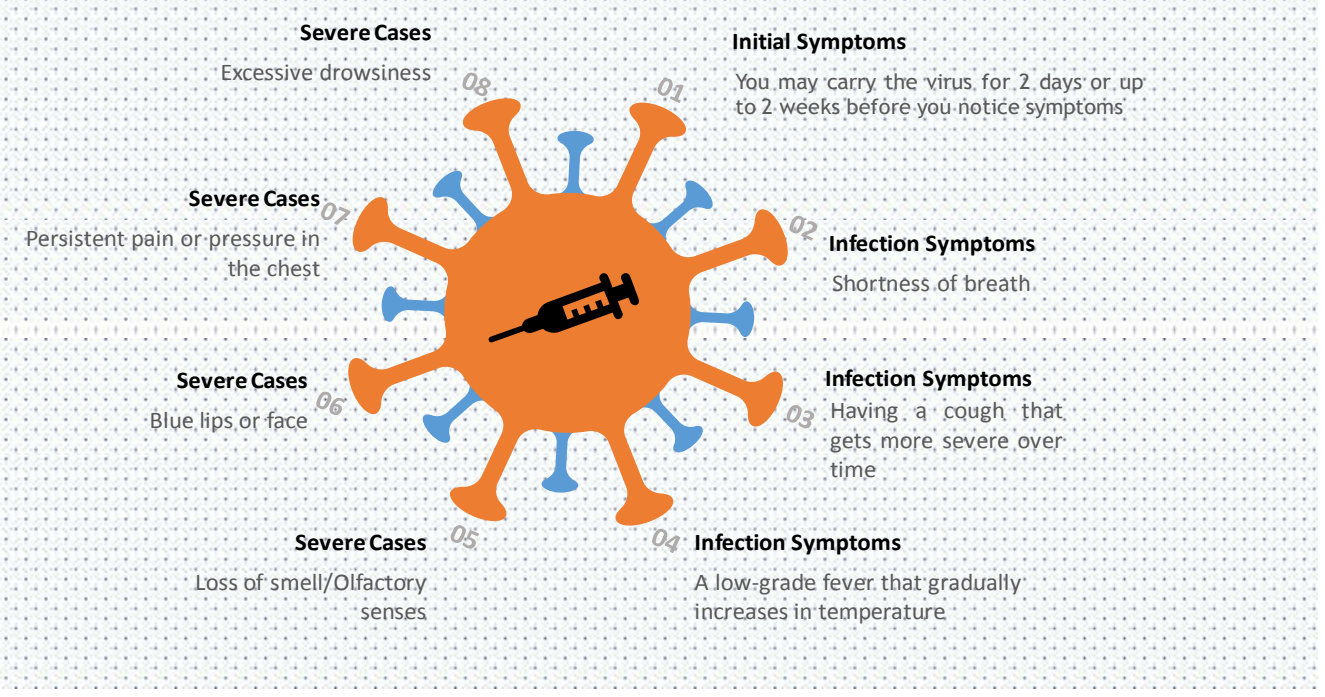

Fig 4: various common symptoms associated with COVID-19

\section{COVID-19 in pregnant women}

However, in case of pregnant women clinical characteristics of COVID-19 were similar to nonpregnant adult patients who developed COVID-19 pneumonia. Pregnant women are more susceptible towards respiratory pathogens and severe pneumonia ${ }^{5}$, because they are at an immunosuppressive state, and physiological adaptive changes. In year 1918 influenza pandemic caused a mortality rate of $2.6 \%$ in the overall population, but $37 \%$ among pregnant women and in COVID-19 no death of pregnant women was reported till date.

\section{Worldwide scenario of COVID 19}

As the world facing new pandemic situation of COVID-19, hence it is important to ensure by the government departments of each country that essential health services and operations to be continued to protect the lives of people with or without COVID 19. Apart from this, it is also important to ensure to avoid spreading misinformation which creates confusion and disturbance among people. To keep this in mind WHO has launched two-way communication network named Information Network for Epidemics (EPI-WIN) website: www.who.int/epi-win on $24^{\text {th }}$ March 2020 which enables the people to access accurate and timely information regarding COVID- $19^{6}$. As per the data received from EPI-WIN, cases and number of deaths is still going on worldwide due to COVID- 19 infection and the major countries are China, The Republic of Korea, Australia, Spain, France, Germany, United Kingdom etc. (Table 1) and (Fig 5 \& 6). 
Table 1: Countries with confirmed COVID-19 cases and deaths. Data as on $25^{\text {th }}$ March 2020*

\begin{tabular}{|c|c|c|}
\hline Reporting Country & Total confirmed cases & Total deaths \\
\hline China & 81848 & 3287 \\
Republic of Korea & 9137 & 126 \\
Australia & 2252 & 8 \\
Philippines & 552 & 35 \\
Malaysia & 1624 & 16 \\
Italy & 69176 & 743 \\
Spain & 39673 & 1100 \\
France & 22025 & 149 \\
Germany & 31554 & 422 \\
India & 8081 & 914 \\
The United Kingdom & 562 & \\
\hline
\end{tabular}

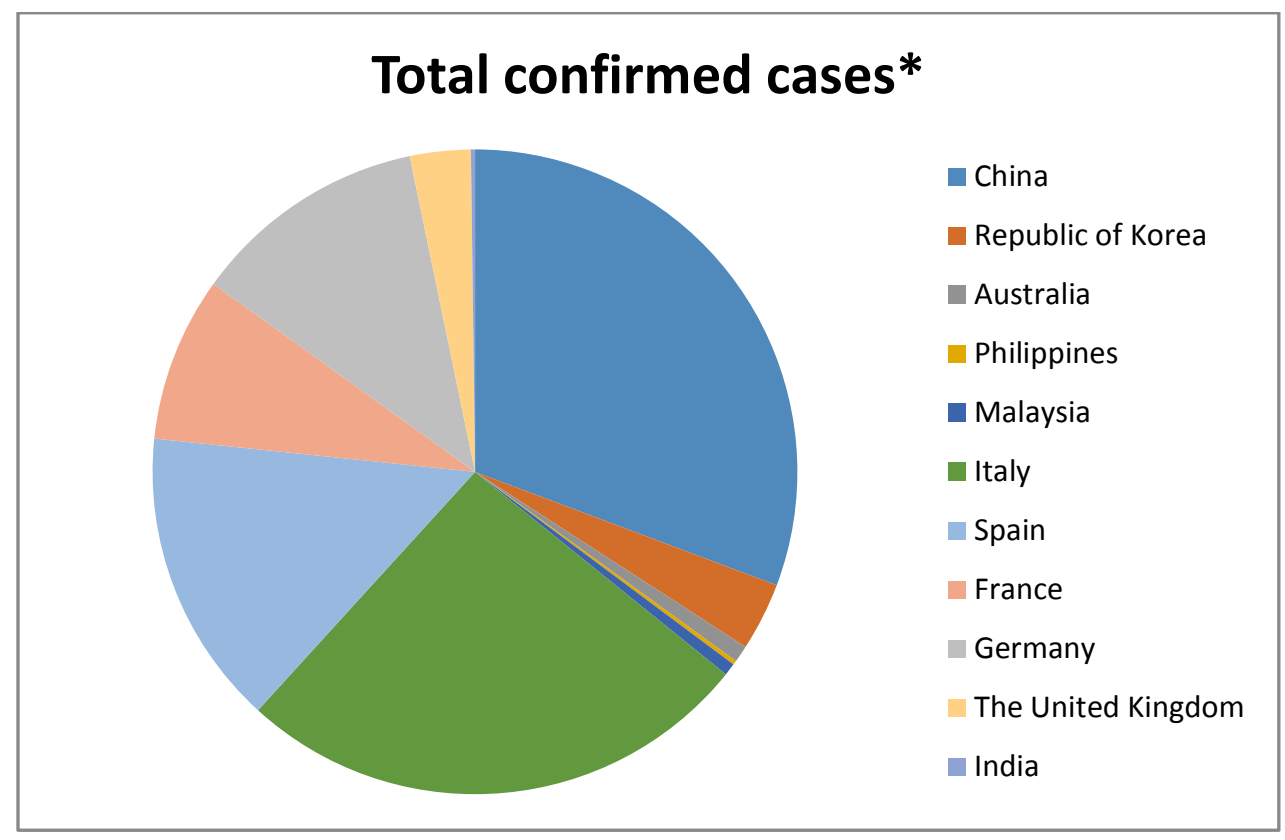

Fig 5: countries with confirmed COVID-19 cases as on 25 March 2020 


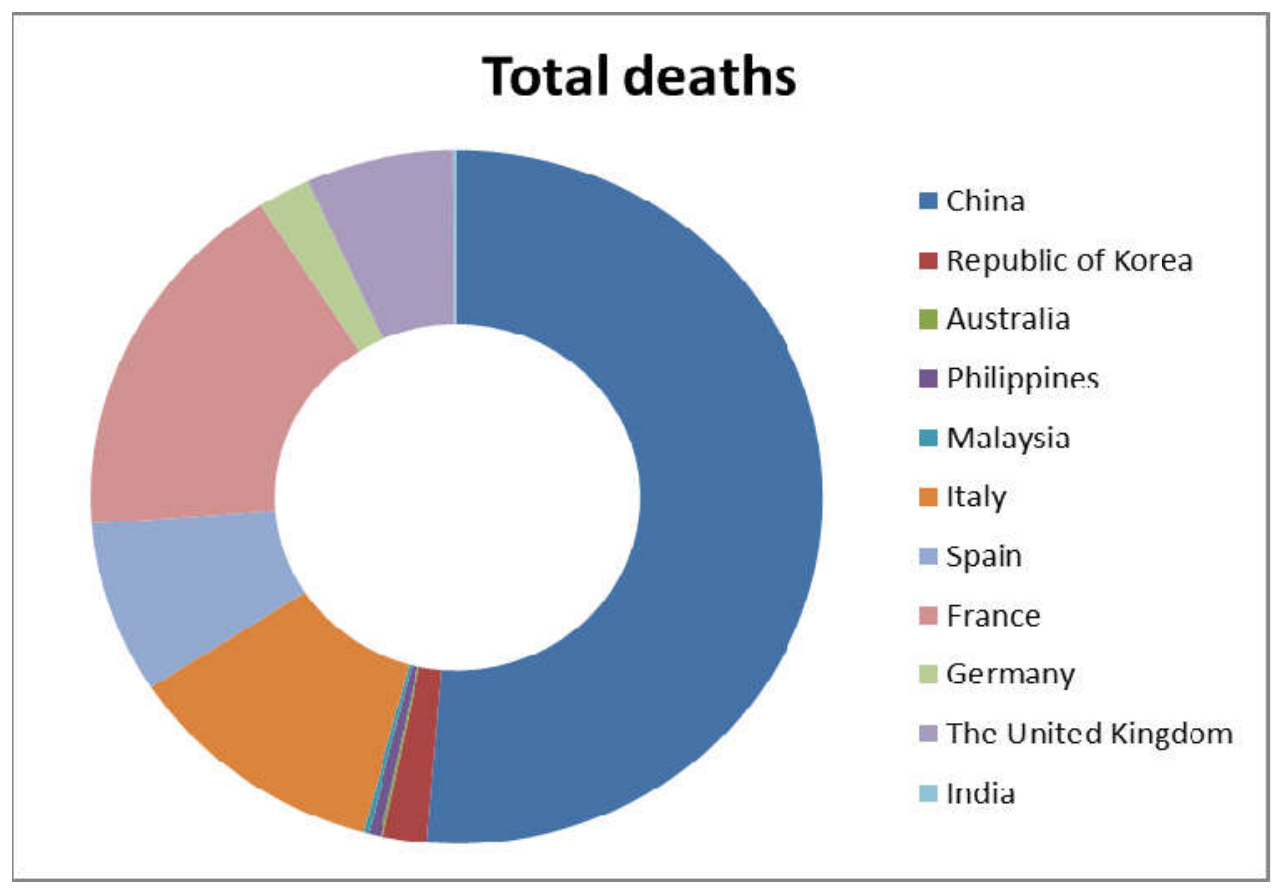

Fig 6: countries with confirmed COVID-19 deaths as on 25 March 2020

\section{Clinical findings}

1. Impact of Age: Individuals of any age can acquire severe COVID 19 infection, although adults of middle age and older are most commonly affected.

2. Laboratory Findings: Leukopenia (most common), leukocytosis, and lymphopenia have been reported, elevated level of lactate dehydrogenase, ferritin and aminotransferase were also observed with variation in white blood cell count.

3. Imaging Findings: COVID-19 most commonly demonstrates ground-glass opacification with or without consolidative abnormalities, consistent with viral pneumonia.

\section{EVALUATION AND DIAGNOSIS}

Earlier COVID-19 can be diagnosed in patients similarly to other conditions caused by viral infections: using a blood, saliva, or tissue sample. However, most tests use a cotton swab to retrieve a sample from the inside of your nostrils. Patients with fever and/or respiratory ${ }^{2}$ tract symptoms (cough, dyspnea) should be considered primarily suspect of COVID-19, but later on patients with severe lower respiratory tract illness and traveller within the prior 14 days to a location are the primary suspect. However, proper microbiologic testing is still going on and researchers decided their priority depending upon the condition given below:

Highest priority patients are:

* Critically ill patients with unexplained viral pneumonia or respiratory failure.

* Any individual with fever or lower respiratory tract illness and close contact with patients confirmed COVID-19 within 14 days of symptom.

* Any individual with fever or lower respiratory tract illness and travel within 14 days of symptom onset to geographic areas with sustained community transmission.

* Individuals with fever or lower respiratory tract illness who are also immune suppressed (including patients with HIV) older.

* Individuals with fever or lower respiratory tract illness who are critical to the pandemic response, including health care workers, public health officials, and other essential leaders.

Second priority includes hospitalized patients, with unexplained fever and lower respiratory tract illness. Third priority includes patients who meet criteria for influenza testing; testing of outpatient pregnant women and symptomatic children with similar risk factors is also included in this priority 
level. Fourth-priority includes patients on community surveillance.

\section{Who is at higher risk}

You're at high risk for contracting SARS-CoV-2, if you come into contact with someone who's carrying it, especially if you've been exposed to their saliva or been near them when they've coughed or sneezed.

$>$ Without taking proper prevention measures, like cover your mouth with mask, sanitize your hand after touching anything and before eating something, avoid social gathering and maintain social distancing etc.

$>$ Live with someone who has contaminated with virus.

> Have an intimate partner who has contracted the virus.

$>$ Lung conditions, such as COPD and asthma.

$>$ Certain heart conditions and immune deficiency conditions, such as HIV, Cancer etc. Severe obesity and other health conditions such as diabetes, kidney disease, or liver disease, if not well-controlled.

\section{What causes corona virus?}

Earlier researchers believe that the virus may zoonotic in nature and first develop in animals either snakes or pangolins before transmitted to humans via open food market in Wuhan, China. Once the virus develops in human, it can be spread by wet stuff method from person to person through respiratory droplets. The viral material hangs out in these droplets and can be breathed into the respiratory tract (your windpipe and lungs), where the virus can then lead to an infection.

\section{TREATMENT}

Unfortunately, currently no treatment available for the COVID-19, however day and night researchers and other healthcare professional performing randomized controlled trials, in humans worldwide before potential vaccines and other treatments become available but this may take several months or longer. Some treatment options that are currently being investigated ${ }^{6}$ by scientists for providing protection against COVID19 are given below:

1. Remdesivir is an experimental broad-spectrum antiviral drug originally designed to target Ebola. This treatment is not yet approved in humans, but two clinical trials for this drug have been implemented in China. One clinical trial was recently also approved by the FDA in the United States.

2. Chloroquine is a drug that's used to fight malaria and autoimmune diseases. It's been in use for more than 70 years and is considered safe.

3. Lopinavir and ritonavir are sold under the name Kaletra and are designed to treat HIV. According to the World Health Organization (WHO), there could be benefits of using Kaletra in combination with other drugs.

4. China has approved the use of the antiviral drug favilavir to treat symptoms of COVID-19. The drug was initially developed to treat inflammation in nose and throat.

5. Hydroxychloroquine alone or in combination with azithromycin may reduce detection of SARS-CoV2 RNA in upper respiratory tract specimens compared with a non-randomized control group but did not assess clinical benefit. Hydroxychloroquine and azithromycin associated with QT prolongation and caution is advised when considering these drugs in patients with chronic medical conditions or who are receiving medications that might interact to cause arrhythmias.

6. Hydroxychloroquine and Chloroquine are orally prescribed drugs that have been used for treatment of malaria and certain inflammatory conditions. Both drugs have in-vitro activity against SARSCoV, SARS-CoV-2, and other Coronaviruses, with Hydroxychloroquine having relatively higher potency against SARS-CoV-2.

\section{CONCLUSIONS}

Latest literature and reports received daily from the official sites worldwide revealed the severity of COVID-19, causing more deaths and increasing number of patients all over the world. However, on the basis of ongoing global public health emergency, our conclusions are limited and we believe that the twophase division first immune defence and second inflammation-driven damaging phase along with above ideas can help combat this deadly contagious disease.

\section{ACKNOWLEDGEMENTS}

The authors are thankful to World Health Organization (WHO) and Indian Council of Medical Research (ICMR), India for providing live updated data on COVID-19. 


\section{REFERENCES}

1. D. Wang, B. Hu, C. Hu, F. Zhu, X. Liu, J. Zhang, et al., Clinical Characteristics of 138 Hospitalized Patients With 2019 Novel Coronavirus-Infected Pneumonia in Wuhan, China. JAMA, 2020; 323:1061-1069.

2. S. Prasad, V. Potdar, S. Cherian, P. Abraham, A. Basu, Transmission electron microscopy imaging of SARS-CoV-2, Ind. J. Med. Res. 2020; DOI: 10.4103/ijmr.IJMR;577-20RI.

3. J. Wang, B. Cao, C. Wang, Science in the fight against the novel coronavirus disease, Chin. Med. J. 2020; DOI: 10.1097/CM9.0000000000000777.
4. N. Zhu, D. Zhang, W. Wang, et al., A novel coronavirus from patients with pneumonia in China, 2019, N Engl J Med., 2020; 382:727-733.

5. H. Chen, J. Guo, C. Wang, F. Luo, X. Yu, Zhang, et al., Clinical characteristics and intrauterine vertical transmission potential of COVID-19 infection in nine pregnant women: a retrospective review of medical records, The Lancet 2020; 395:809-815.

6. M. Cascella, M. Rajnik, A. Cuomo, S. C. Dulebohn, R. D. Napoli, Features, Evaluation and Treatment Coronavirus (COVID-19), Stat Pearls (internet) 2020; [PubMed: 19430490]. 